\title{
RATCHET EFFECT FOR NANOPARTICLE TRANSPORT IN HAIR FOLLICLES
}

\author{
MATTHIAS RADTKE, ALEXA PATZELT, FANNY KNORR, JÜRGEN LADEMANN, \\ AND ROLAND R. NETZ
}

\begin{abstract}
The motion of a single rigid nanoparticle inside a hair follicle is investigated by means of Brownian dynamics simulations. The cuticular hair structure is modeled as a periodic asymmetric ratchet-shaped surface. Induced by oscillating radial hair motion we find directed nanoparticle transport into the hair follicle with maximal velocity at a specific optimal frequency and an optimal particle size. We observe flow reversal when switching from radial to axial oscillatory hair motion. We also study the diffusion behavior and find strongly enhanced diffusion for axial motion with a diffusivity significantly larger than for free diffusion.
\end{abstract}

\section{INTRODUCTION}

Hair follicles constitute an important pathway for topically applied drug formulations used in medical applications to overcome the skin barrier $[1,2]$. In modern drug delivery applications, often nanoparticles are used as carrier systems [3]. It has been shown that solid nanoparticles can effectively penetrate into the hair follicles [4], however, typically they do not penetrate all the way into the viable epidermis $[5,6]$. The follicle thus can serve as a reservoir for nanoparticles loaded with drugs, and after release drug molecules may translocate the skin barrier and be delivered to specific target sites $[7,8]$.

Experiments are typically performed in vitro on porcine ear skin as a common model for human skin [2]. Enhanced particle penetration was demonstrated after massaging the skin, the main effect of which presumably is to mechanically move the hair relative to the follicle, similar to the omnipresent motion of hair in typical every-day-live situations [9]. It was suggested that moving hair acts as a ratchet, which by its oscillatory motion drives the nanoparticles deeply into the hair follicles. Further investigations revealed a strong dependence of the transport efficiency on the nanoparticle size [10]. The optimal particle size of about $600 \mathrm{~nm}$ corresponds to the thickness of overlapping cuticular hair surface cells [9]. Since the hair structure resembles a sawtooth-like profile with a rather well defined corrugation amplitude, a ratchet mechanism indeed might explain the enhanced particle transport induced by hair motion. Other material properties and the solvent properties were shown to only have a minor influence on the transport efficiency of nanoparticles [11].

Date: October 7, 2016. 
The so-called ratchet effect has been amply described in literature and refers to directed particle motion generated by a non-equilibrium perturbation of a periodic system. One necessary ingredient is that the spatial symmetry is broken in order to single out a direction [12-15]. Ratcheting surfaces are observed in a number of different biological systems ranging from biopolymers such as actin [16] to macroscopic systems like plants [17].

In this study we propose a simple two-dimensional stochastic model for single nanoparticle transport inside a moving hair follicle. Our model incorporates the periodic asymmetric hair structure and the oscillatory hair motion, which mimics the effect of skin massage or other external forces acting on the hair. We show that these ingredients indeed lead to directed nanoparticle motion. For varying frequency of radial hair motion perpendicular to the hair axis, we find directed motion into the hair follicle with an optimal driving frequency. We also find a slight enhancement of the diffusivity. We observe reversal of the transport direction when changing the mode of hair movement from radial to axial motion parallel to the hair axis. For axial motion, a significant enhancement of the diffusion is observed with diffusivities larger than for free diffusion. By introducing an effective viscosity, our directed ratchet model can explain the large follicular penetration depths observed in experiments on nanoparticle transport into pig skin under applied massage. Studying the influence of the particle size on the transport efficiency, we determine, in agreement with experiments, an optimal particle size. The first section introduces our model, discusses the model simplifications and explains the Brownian dynamics simulations. In the last section we give a short conclusion and discuss future work.

\section{Model AND Simulation Details}

The motion of a single nanoparticle inside a moving, structured hair follicle is modeled as a random walk of a rigid particle in two dimensions that is interacting with the surface of the hair and the follicle surface. As schematically depicted in fig. 1a, we use the simplistic description of an asymmetric and fully periodic sawtooth structure to represent the cuticular structure of hair, shown in fig. 1b, while the inner surface of the follicle is modeled as a completely smooth surface. The sawtooth structure of the hair, shown in fig. 1a in black, is characterized by the corrugation amplitude $\chi$ (cuticular thickness) and the periodicity $L$. For sake of simplicity and speed of our simulations, both the hair surface and the follicle surface are in our model assumed to be infinitely rigid. Note that the rigid nanoparticle is in fact modeled as a point-like particle. The effect of different particle sizes is taken into account in our simulations via the effective distance $d$ between hair and follicle. In order to connect to the experimental scenario, the experimental distance is given by $d_{\exp }=d+2 R$ and thus implicitly incorporates the effect of changing particle radius $R$.

We model the steric repulsion of the nanoparticle from the two surfaces, which are separated by a distance $d$, employing exponentially decaying repulsive potentials that are located on the hair surface and the follicle surface

$$
\begin{aligned}
& U_{h}(x)=e^{-\frac{x+d / 2-A_{x} \sin \omega t}{\kappa}} \\
& U_{f}(x)=e^{\frac{x-d / 2}{\kappa}}
\end{aligned}
$$


a)

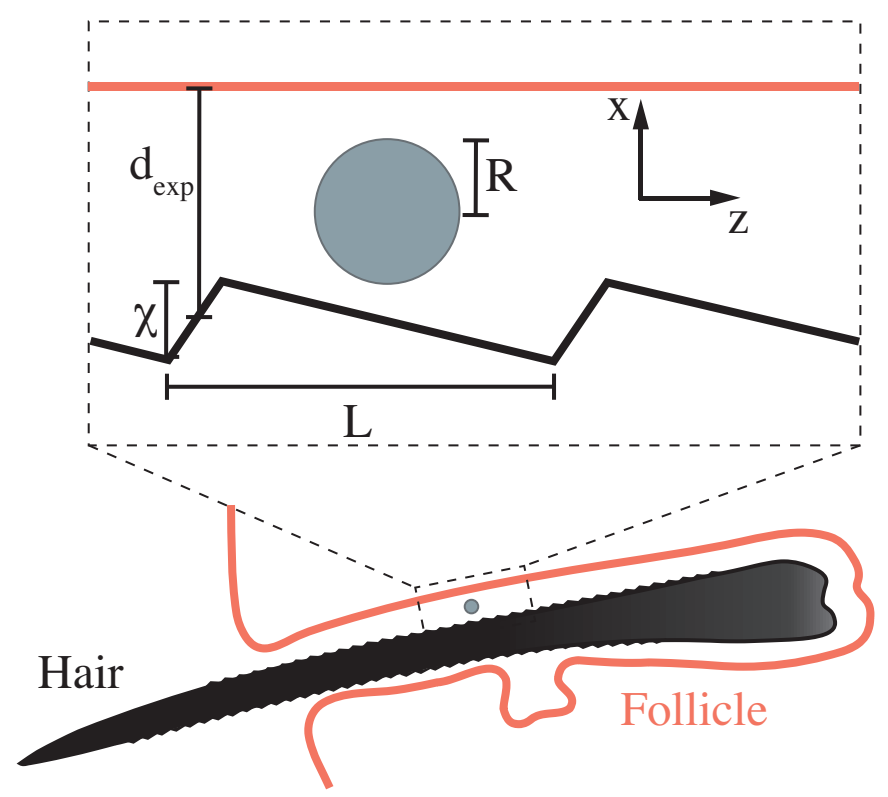

b)

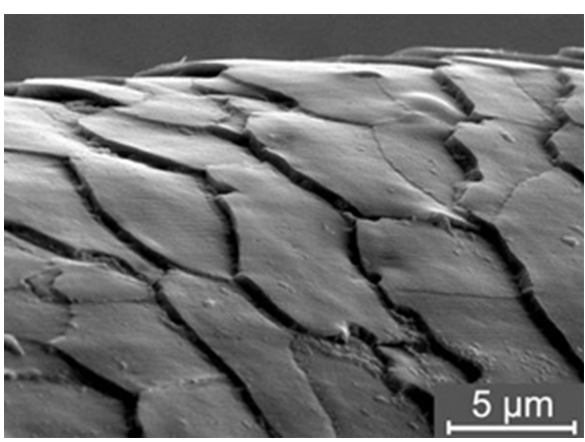

c)

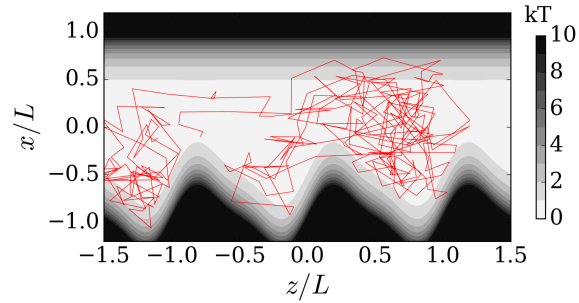

Figure 1. a) Schematic illustration of the model geometry and the model parameters. A single hair is located inside the follicle. A single rigid nanoparticle of radius $R$ (grey) is present in the space between hair and follicle and interacts with the surface of the hair (shown in black) and the surface of the follicle (shown in red). Both surfaces are separated by a distance $d_{\text {exp }}$. The cuticular hair surface is represented by a sawtooth structure with a corrugation height $\chi$ and a periodicity $L$. b) Electronmicroscope image of the cuticular structure of a human terminal hair, which serves as a motivation for our simplified geometric model [18]. c) Contour plot of the potential that mimics the hair and follicle surfaces and confines the nanoparticle, according to eq. (4) in units of $\mathrm{kT}$ and for the rescaled model parameters $\left(d_{\exp }+2 R\right) / L=1.0, \chi / L=0.3$. The red trajectory illustrates the Brownian random motion of the nanoparticle center for the case of motionless hair.

with a short rescaled surface interaction range $\kappa / L=0.2$.

Since it is not clear how a mechanical perturbation acting on the external hair section translates into the relative motion of the hair with respect to the follicle, we study a general model and separately investigate the effects of radial and axial hair motion. Radial hair motion perpendicular to the hair axis is modeled via the time-dependent periodic term in the potential eq. (1) with frequency $\omega$ and amplitude $A_{x}$. The resulting motion of the hair surface in $\mathrm{x}$-direction is illustrated in the top of fig. $2 \mathrm{~b}$. The sawtooth 
shape of the hair surface is modeled with a ratchet-shape function [19] given by

$$
F(z)=-\chi\left[\sin (2 \pi z / L)+\frac{1}{4} \sin (4 \pi z / L)\right] .
$$

The total potential energy acting on the nanoparticle reads

$$
U(x, z)=U_{f}(x)+U_{h}\left(x+F\left(z+A_{z} \sin \omega t\right)\right),
$$

where we introduce the axial periodic motion of the hair with respect to the follicle with amplitude $A_{z}$, which is illustrated in the bottom of fig. $2 \mathrm{~b}$. A contour plot of the two-dimensional potential energy landscape, eq. (4), is shown in fig. 1c for rescaled parameters $d / L=1.0$ and $\chi / L=0.3$, the red line is a simulated Brownian trajectory of a nanoparticle in the absence of hair motion.

We perform Brownian dynamics simulations based on the overdamped two-dimensional Langevin equation,

$$
\begin{aligned}
& \dot{x}=-\mu_{0} \partial_{x} U(x, z)+\xi_{x}(t) \\
& \dot{z}=-\mu_{0} \partial_{z} U(x, z)+\xi_{z}(t),
\end{aligned}
$$

an example of a resulting nanoparticle trajectory is given in fig. 1c. The first term in eq. (5) accounts for the direct force acting on the particle, which is proportional to the nanoparticle mobility $\mu_{0}$. The second term represents the stochastic contribution $\xi_{\alpha}$ given by Gaussian random components with correlations according to the fluctuation-dissipation theorem, $\left\langle\xi_{\alpha}(t) \xi_{\beta}\left(t^{\prime}\right)\right\rangle=2 \mathrm{kT} \mu_{0} \delta_{\alpha \beta} \delta\left(t-t^{\prime}\right)$, and vanishing mean [20]. In the following, all parameters and quantities are made dimensionless by rescaling lengths according to $\tilde{x}=x / L$ by the periodic length scale of the sawtooth structure $L$, energies $\tilde{U}=U / \mathrm{kT}$ by thermal energy and times $\tilde{t}=t / \tau$ by the characteristic particle diffusion time $\tau=L^{2} / \mu_{0} \mathrm{kT}=6 \pi \eta R L^{2} / \mathrm{kT}$ with the viscosity $\eta$. For one set of parameters, the simulation typically runs for $10^{9}$ time steps with a rescaled time step $\Delta t / \tau=10^{-4}$. From the simulations we obtain the average axial velocity $V=<\left(z\left(t+10^{3} \Delta t\right)-z(t)\right) /\left(10^{3} \Delta t\right)>$ by sampling the particle position every $10^{3}$ steps. The axial diffusivity $D$ then follows by calculation of the mean square displacement according to

$$
<(z(t)-z(0))^{2}>=2 D t+V^{2} t^{2} .
$$

\section{RESUlts}

3.1. Transport properties of a particle under radial and axial hair motion. First we study the particle transport for periodic hair motion that is radial with respect to the follicle as a function of driving frequency $\tilde{\omega}$ with fixed motion amplitude $\tilde{A}_{x}=0.5$ (while $\tilde{A}_{z}=0$ ). This situation is schematically depicted in the top of fig. $2 \mathrm{~b}$. The distance $\tilde{d}=0.2$ between hair and follicle surface is fixed and the corrugation amplitude is set to $\tilde{\chi}=0.5$. Results for the average velocity $\tilde{V}$ and diffusivity $\tilde{D}$ are shown in fig. 2a,c as black symbols. For radial hair motion, we find enhanced particle transport into the hair follicle, as seen by the positive average velocity in fig. 2a as a function of frequency. There is an optimal frequency $\tilde{\omega} \approx 100$ at which the average velocity exhibits a maximum. As shown in fig. 2c, the diffusivity $\tilde{D}$ exhibits a maximum at somewhat smaller values of $\tilde{\omega}$ compared to the frequency at which the average velocity is maximal. 
a)

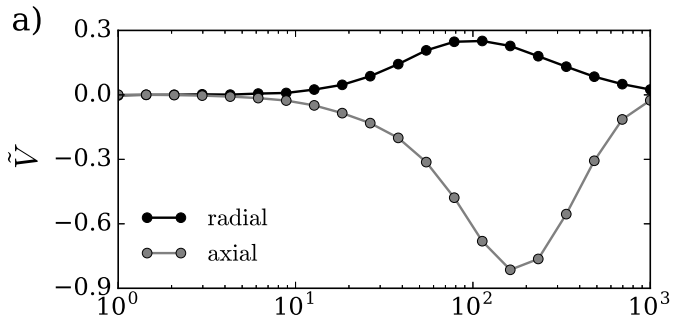

C)

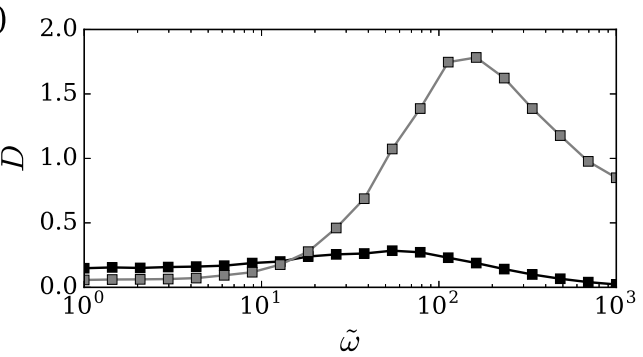

b)
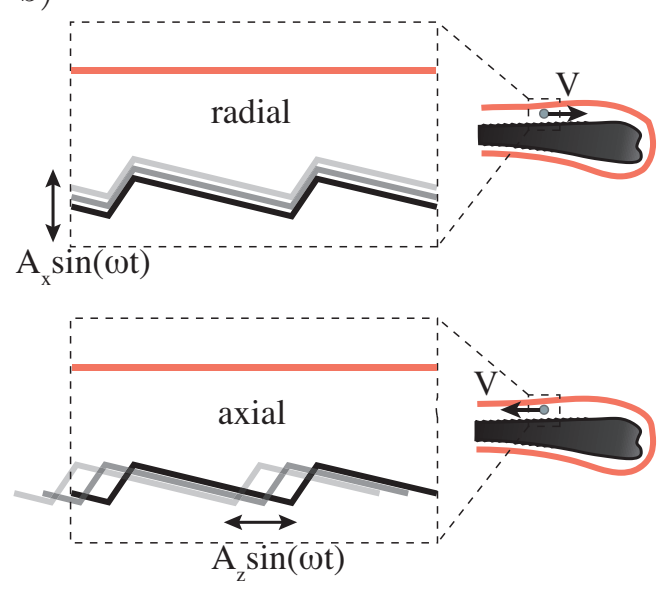

FiguRE 2. Results for nanoparticle motion induced by radial (black symbols) and axial (gray symbols) hair motion with amplitudes $\tilde{A}_{x}=0.5$ (while $\tilde{A}_{z}=0$ ) and $\tilde{A}_{z}=0.5$ (while $\tilde{A}_{x}=0$ ), respectively. The corrugation amplitude of the hair surface is fixed to $\tilde{\chi}=0.5$ and the distance between the hair and follicle surfaces is $\tilde{d}=0.2$. a) For radial motion the average rescaled velocity $\tilde{V}$ is positive (i.e. into the follicle) and increases with increasing frequency up to a maximum at $\tilde{\omega} \approx 100$. In contrast, for axial motion $\tilde{V}$ is negative (i.e. out of the follicle) and with increasing frequency exhibits a minimum at $\tilde{\omega} \approx 150$. b) Illustration of the radial (top) and axial (bottom) modus of hair motion and the resulting transport into or out of the follicle, respectively. c) The diffusivity $\tilde{D}$ as a function of frequency for radial motion has a weak maximum at smaller frequencies $\tilde{\omega} \approx 50$ than the maximum in the average velocity $\tilde{V}$ seen in a). Axial hair motion leads to a pronounced maximum in diffusivity at a frequency corresponding to the minimum in average velocity shown in a).

Around the maximum, the diffusivity slightly exceeds the equilibrium $(\tilde{\omega}=0)$ value of $\tilde{D}_{0}=0.05$.

Next we consider axial motion of the hair with amplitude $\tilde{A}_{z}=0.5$ (while $\tilde{A}_{x}=0$ ), as schematically shown in the bottom of fig. $2 \mathrm{~b}$. The average velocity and diffusivity as a function of frequency $\tilde{\omega}$ are presented as gray symbols in fig. 2a,c for fixed $\tilde{\chi}=0.5$ and distance $\tilde{d}=0.2$. As opposed to radial hair motion, we observe a negative velocity, that means directed particle transport out of the hair follicle. The velocity decreases with increasing frequency to a minimum at about $\tilde{\omega}=150$. Simultaneously, the diffusivity shown in fig. 2c exhibits a pronounced maximum. Diffusivities $\tilde{D}>1$ larger than for free diffusion are observed, which is a substantial enhancement compared to the equilibrium case, corresponding to the limit $\tilde{\omega}=0$, for which $\tilde{D}_{0}=0.05$. We conclude that axial hair 
motion can greatly enhance the diffusive transport of the nanoparticle but also leads to directed transport out of the follicle. This raises the question what the dominating mode of transport for axial hair motion is, a question we will address later on.

In fig. 3 we give a pictorial explanation for the difference between radial and axial hair motion and the resulting flashing and pushing ratchet effects, respectively. Radial hair motion, illustrated in fig. 3a, causes an effect similar to what is known as flashing ratchet effect [14]. In contrast to previous theoretical models, here we consider a moving ratchetshaped surface and two-dimensional particle motion instead of a ratchet potential in one dimension that is switched on and off. However, the mechanism of alternating particle localization and diffusion over a barrier is similar, including the necessary condition of a spatial symmetry breaking of the potential landscape. As seen in fig. 3a, radial hair motion leads to particle transport to the right which can be understood by the fact that particles in step i) are pushed down the shallow flank to the right and in step ii) diffuse from the minima over the steep flank. Axial hair motion, illustrated in fig. 3b, causes a pushing ratchet effect. Due to the spatial asymmetry of the potential, particles are in step i) predominantly pushed to the left by the steep flank and in step ii) diffuse over the barrier of the shallow flank to the next minimum on the left.
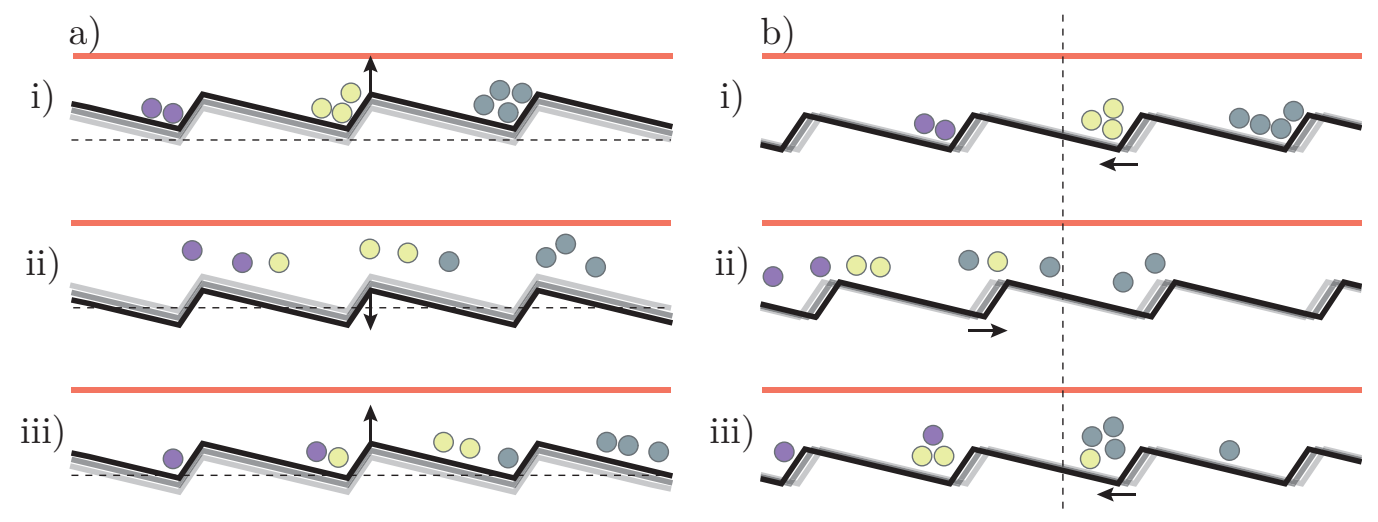

FiguRE 3. a) Illustration of radial hair motion and the flashing ratchet effect that leads to transport of the nanoparticles to the right, i.e. down the shallow slope. i) Upward hair motion leads to strong particle localization in the potential minimum. Subsequent downward motion in ii) allows relaxation and particle diffusion from the minima over the barrier with the steep slope. Transport to the right results in iii) from upwardly moving hair that pushes some particles down the shallow slope. b) Illustration of the axial hair motion and the pushing ratchet effect that leads to transport of the nanoparticles to the left. i) The hair moves to the left and the steep slope pushes particles to the left. ii) Hair motion to the right has a less pronounced effect on the particles, which can diffuse over the flank with the shallow slope to the left. iii) Subsequent leftward hair motion again leads to localization and forced motion of the particles to the left. 
3.2. Recovering physical dimensions. In order to compare our simulation results with experimental results, we present in fig. 4a,b the average nanoparticle velocity as a function of radial driving frequency in physical units, using the same data as already shown in fig. 2a. We assume a characteristic corrugation length scale $L=5 \mu \mathrm{m}$, i.e. the actual length scale of the cuticular hair structure in fig. 1a, the corrugation amplitude $\chi=2.5 \mu \mathrm{m}$, and a particle radius $R=0.3 \mu \mathrm{m}$. In experiments, nanoparticles with radii between $R=0.06-0.5 \mu \mathrm{m}$ were tested and most efficient transport was found for $R \approx 0.32 \mu \mathrm{m}[10]$. Simulation results are shown for temperature $T=310 \mathrm{~K}$ and two different viscosities: in fig. $4 \mathrm{a}$ for the viscosity of water, $\eta=0.001 \mathrm{Pas}$, and in fig. $4 \mathrm{~b}$ for the viscosity of human sebum, $\eta=0.065 \mathrm{Pas}$ [21]. For the viscosity of water, a maximal particle velocity of $V=38 \mathrm{~nm} / \mathrm{s}$ is reached at a frequency of about $\omega=3 \mathrm{~s}^{-1}$, while for the viscosity of human sebum a maximal particle velocity of $V=0.6 \mathrm{~nm} / \mathrm{s}$ is reached at a much lower frequency of about $\omega=0.04 \mathrm{~s}^{-1}$. We conclude that the local viscosity inside the hair follicle is a very important parameter that sensitively influences the directed particle transport properties.

In order to compare with the experimental penetration depth $l_{\exp }=300 \mu \mathrm{m}$ obtained in the absence of massage and the value of more than $l_{\exp }=1000 \mu \mathrm{m}$ after 3 min massage and $30 \mathrm{~min}$ penetration time [4], in the following we define an effective viscosity $\eta_{\text {eff. }}$. The estimated experimental diffusivity without massage follows from the measured penetration depth $l_{\exp }=300 \mu \mathrm{m}$ for penetration time $t_{\exp }=30 \mathrm{~min}$ as $D_{0}^{\exp }=l_{\exp }^{2} / t_{\exp }=50 \mu \mathrm{m}^{2} / \mathrm{s}$. In our model we measure a rescaled equilibrium diffusivity of $\tilde{D}_{0}=0.05$. By setting $D_{0}=D_{0}^{\exp }$, we obtain the effective viscosity $\eta_{\mathrm{eff}}=\frac{\tilde{D}_{0} \mathrm{kT}}{6 \pi D_{0}^{\mathrm{exp}} R} \approx 0.8 \times 10^{-6} \mathrm{~Pa}$ s, which is much smaller than water. This could mean that the space between hair and follicle surface is only partially filled with liquid or that chemical potential gradients pull particles into the follicle. Using this effective viscosity, we reach a maximal particle velocity with massage of $V=46.1 \mu \mathrm{m} / \mathrm{s}$ and thus a penetration depth after 3 min massage of about $8300 \mu \mathrm{m}$, even larger than the experimental value of $l_{\exp }=1000 \mu \mathrm{m}$.

We conclude that by introducing an effective viscosity that is even much smaller than water, the directed ratchet transport model is able to explain the large penetration depth observed in experiments. However, it remains unclear whether also the diffusion enhancement is a relevant factor for the nanoparticle transport.

In order to illustrate the difference between directed and diffusive transport, we plot in fig. 4c the characteristic time scale for directed motion, defined by $t_{v}=l /|V|$, and the characteristic time scale for diffusive motion, defined by $t_{d}=l^{2} / D$, as a function of penetration depth $l$ using the maximal velocity $V=38 \mathrm{~nm} / \mathrm{s}\left(\omega=3.4 \mathrm{~s}^{-1}\right)$ from fig. 4a, where the diffusivity is $D=0.17 \mu \mathrm{m}^{2} / \mathrm{s}$. For comparison, the time scale for diffusion in the absence of hair motion $(\omega=0) t_{0}=l^{2} / D_{0}$ is shown as a dotted line for the measured value $D_{0}=0.04 \mu \mathrm{m}^{2} / \mathrm{s}$. It is seen that hair motion significantly enhances diffusion and thus decreases the diffusion time. For short times less than about $120 \mathrm{~s}$, diffusive transport, shown as a dashed line in fig. $4 \mathrm{c}$, is more effective, that means the diffusive time scale $t_{d}$ is smaller compared to directed transport shown as a solid line. At a penetration depth $l^{*}=D / V$, indicated by a vertical gray bar in fig. 4c, both time scales are equal, $t_{v}=t_{d}$. For larger times $t>120 \mathrm{~s}$ directed motion is the important mode of 
a)

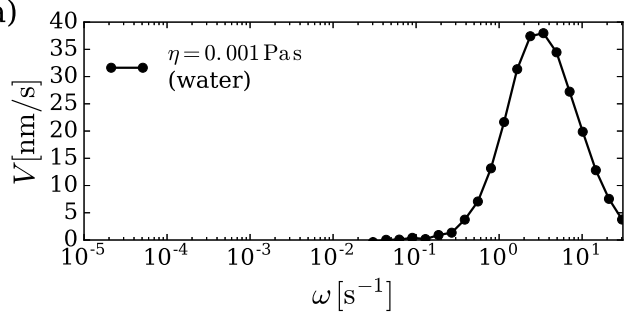

b)

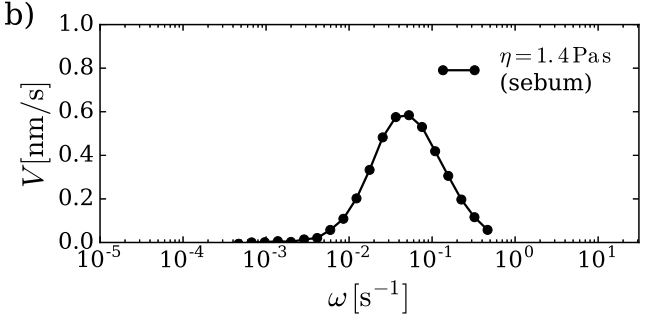

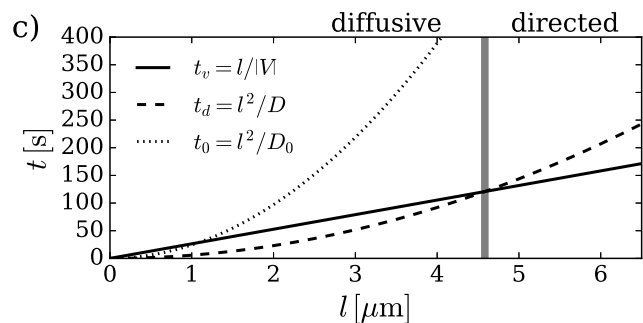

d)

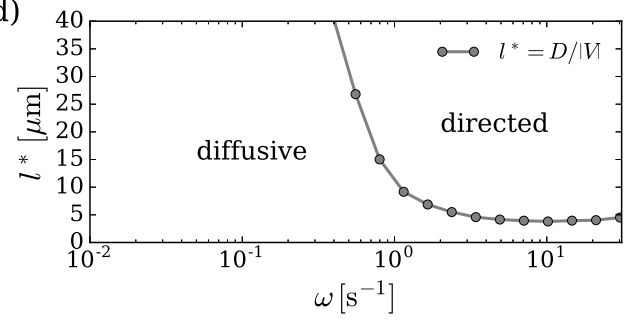

FIgURE 4. Average transport velocity $V$ in physical units as a function of radial driving frequency $\omega$ for fixed parameters $L=5 \mu \mathrm{m}, \chi=2.5 \mu \mathrm{m}, R=$ $0.3 \mu \mathrm{m}, d=1 \mu \mathrm{m}, A_{x}=2.5 \mu \mathrm{m}\left(A_{z}=0\right), T=310 \mathrm{~K}$, and two different viscosities for a) water $\eta=0.001 \mathrm{Pas}$ and b) sebum $\eta=0.065 \mathrm{Pas}$. c) Comparison of the characteristic time scale for directed motion $t_{v}=l /|V|$ (solid line) and diffusive motion $t_{d}=l^{2} / D$ (dashed line) as a function of penetration depth $l$ for the viscosity of water and parameters for maximal particle velocity in a): $\omega=3.4 \mathrm{~s}^{-1}, V=38 \mathrm{~nm} / \mathrm{s}$, and $D=0.17 \mu \mathrm{m}^{2} / \mathrm{s}$. The dotted line shows the time scale for diffusive motion in the absence of external driving $(\omega=0)$ for which $D_{0}=0.04 \mu \mathrm{m}^{2} / \mathrm{s}$. The vertical gray bar indicates the transition from diffusive to directed transport. d) The corresponding length $l^{*}=D /|V|$ at which both time scales are equal, $t_{v}=t_{d}$, is plotted as a function of frequency.

transport, leading to larger penetration depths than is achieved by diffusive transport. The crossover from the diffusive regime, i.e. $t_{d}<t_{v}$, to the regime of directed transport, i.e. $t_{v}<t_{d}$, occurs at a penetration depth $l^{*} \approx 5 \mathrm{~nm}$ corresponding to the periodicity $L$ of the cuticular hair structure. In fig. $4 \mathrm{~d}, l^{*}=D /|V|$ is shown as a function of frequency $w$ for the same parameters used in fig. 4a. We conclude that for frequencies larger than about $\omega=1 \mathrm{~s}^{-1}$ the crossover to directed transport occurs at such low penetration depths that in order to explain experimental results, the directed particle motion induced by moving hair is the more important mechanism.

3.3. Influence of particle size. The investigation of the effect of different distances $\tilde{d}$ between the hair and follicle surfaces in our model is a way to determine the influence of particle size on the particle transport efficiency. In fig. 5a,b we show results for the average particle velocity $\tilde{V}$ and diffusivity $\tilde{D}$, respectively, for radial hair motion with fixed frequency $\tilde{\omega}=100$ as a function of $\tilde{d}$. Small distances induce particle trapping between the two surfaces and thus lead to small velocities and small diffusivities. At large surface distances the particle has on average a large separation from the hair surface 
and thus does not feel the hair motion, consequently the ratchet effect vanishes and the particle transport velocity goes to zero. The optimal distance at which particle transport velocity into the follicle is maximal is seen to be $\tilde{d}=0.5$, which turns out to be of the same magnitude as the hair corrugation amplitude $\tilde{\chi}=0.5$. This means that directed transport is most pronounced when the surfaces are close but not overlapping. The diffusivity in fig. 5b monotonically increases with distance $\tilde{d}$. For large distances $\tilde{d}>5$ the particle diffusion is not perturbed by the moving hair surface and thus the free diffusion limit with $\tilde{D}=1$ is approached, as seen in fig. $5 \mathrm{~b}$.

Experimental penetration depths in hair follicles [10] as a function of PLGA (polylactid-co-glycolid) particle size are presented in fig. 5c. It is seen that the nanoparticle penetration into hair follicles is optimal at an intermediate particle diameter of about $600 \mathrm{~nm}$. The relation between the hair-follicle distance used in the simulation model $d$, where the nanoparticle is assumed point-like, and the total hair-follicle distance $d_{\exp }$ for a finite-size particle, is given by $d=d_{\exp }-2 R$. This allows to relate the maximum in transport velocity seen in the simulation data in fig. $5 \mathrm{a}$ as a function of $\tilde{d}=d / L$ with the optimal penetration seen in the experimental data in fig. $5 \mathrm{c}$ as a function of $2 R$. In essence, decreasing the particle diameter $2 R$ at fixed total hair-follicle distance $d_{\text {exp }}$ corresponds to increasing the distance $d=d_{\exp }-2 R$ in the simulation model. We can thus identify the optimal particle diameter of $2 R=643 \mathrm{~nm}$ in the experimental data in fig. $5 \mathrm{c}$ with the optimal distance $d=2500 \mathrm{~nm}$ in the simulation data in fig. $5 \mathrm{a}$, obtained via $d=\tilde{d} L$ assuming a corrugation period of $L=5000 \mathrm{~nm}$. According to our model, the total hair-follicle separation follows to be of the order of $d_{\exp }=d+2 R \approx 3100 \mathrm{~nm}$. Since this separation presumably corresponds to the most highly constricted region in the follicle, this seems to be a realistic value.

\section{Conclusion}

In the present study we demonstrate, using a simple stochastic two-dimensional simulation model, that a ratchet mechanism is able to explain the penetration enhancement of nanoparticles into hair follicles. We distinguish two transport mechanisms: directed motion and enhanced diffusive motion, both are caused by the oscillatory hair motion. We find that radially moving hair causes directed nanoparticle transport into the hair follicle, which is most efficient at an optimal driving frequency and at an optimal particle size. By introducing an effective viscosity, the directed ratchet transport model yields penetration depths even larger than observed in experiments. This might be due to the fact that experiments haven been done at a fixed and most likely sub-optimal frequency of the massage device. We show that a diffusive transport mechanism can also play an important role. We estimate the crossover from diffusive to directed transport for radial hair motion to occur at a penetration depth of the order of $5 \mu \mathrm{m}$, which is much smaller than the experimentally observed penetration depths of more than $1000 \mu \mathrm{m}$. In order to predict the relevance of diffusion for the nanoparticle transport, it would be important to know the amount of applied nanoparticles that actually penetrate into the follicle besides the maximal penetration depth. Further experimental studies at variable frequency would also be highly interesting and in fact are needed be needed in order to 

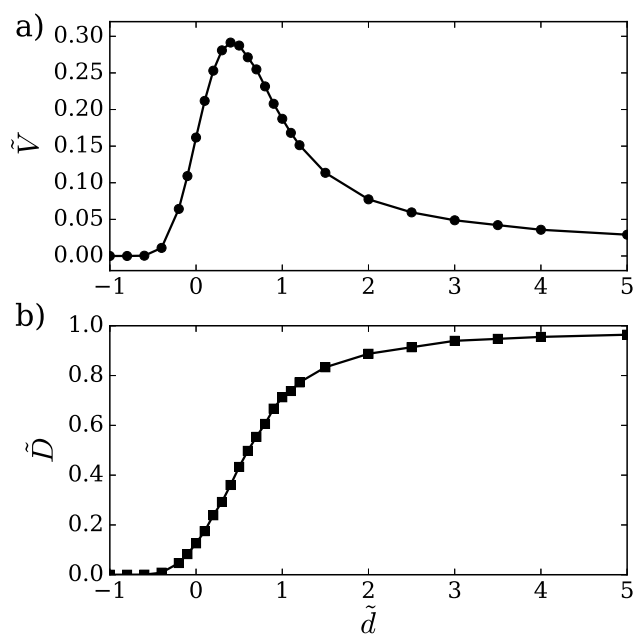

c)

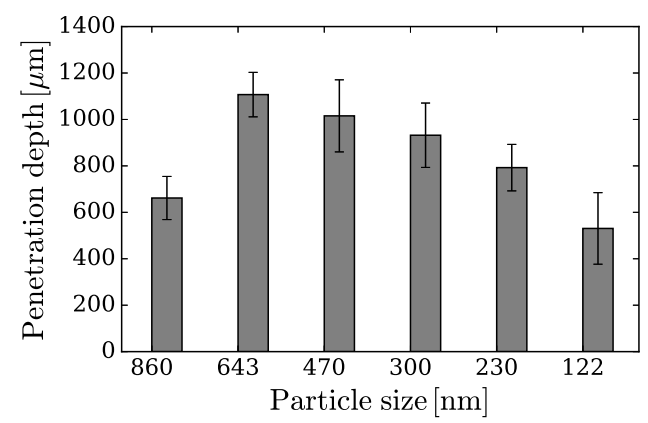

FiguRE 5. Results for a particle under radial hair motion with fixed frequency $\tilde{\omega}=100$ and amplitude $A_{x}=0.5$. a) The average velocity $\tilde{V}$ and the b) diffusivity $\tilde{D}$ is plotted as a function of distance $\tilde{d}$ between the hair and the follicle surface. The optimal distance for enhanced transport of the particle toward the root is about $\tilde{d}=d / L=0.5$ ( $L$ being the corrugation period seen in fig. 1a) at which $\tilde{V}$ exhibits a maximum. The diffusivity in b) monotonically increases toward the free diffusion limit. c) Experimental penetration depths for PLGA particles as a function of particle diameter $2 R[10]$. Decreasing particle diameter corresponds to increasing net hair-follicle distance $d$ in our simulation model.

confirm our prediction about the frequency dependency of the transport efficiency and the existence of an optimal frequency.

In the experimental studies we compare our simulations with, the hair motion is caused by massage [10]. In these experiments, the precise fashion in which the hair is moving relative to the hair follicle is not precisely known and presumably correspond to a superposition of radial and axial oscillatory hair motion. This serves as a motivation for our work in which we study the effects of these two modes of motion separately. In contrast to pure radial hair motion, which causes a flashing ratchet effect and transports nanoparticles into the follicle, pure axial hair motion leads to nanoparticle transport in the opposite direction, i.e. out of the follicle. We denote this transport mechanism as pushing ratchet mode. In this case we observe a significant enhancement of the diffusivity, which even becomes larger than for the free diffusion case. Since particle transport into the hair follicle is observed in the experiment, we speculate that radial hair motion is the dominating mode, but more experiments would be needed to settle this question. In future work it would certainly be interesting to study a more general simulation model and to investigate the effects of the superposition of radial and axial hair motion. 
In conclusion, our simple two-dimensional stochastic model demonstrates the basic mechanism of nanoparticle transport on a ratchet-shaped hair surface as well as the influence of parameters such as driving frequency and particle size. Future model refinements should take into account several other important factors that might influence the nanoparticle transport efficiency. Those include more complicated non-periodic and disordered structures of the follicle and the hair surfaces which might lead to steric hair-follicle interlocking [22]. One other interesting model generalization would include adhesion effects between particles and the hair and follicle surface as well as viscoelastic properties of the liquid medium between hair and follicle [23, 24]. Furthermore, effects of nanoparticle and follicle as well as hair elasticity and inter-particle interactions are important future lines of work.

Financial support from the DFG Collaborative Research Center 1112 - "Nanocarriers: Architecture, Transport and Topical Application of Drugs for Therapeutic Use" is acknowledged.

\section{REFERENCES}

[1] J. Lademann, H. Richter, M.C. Meinke, B. Lange-Asschenfeldt, C. Antoniou, W.C. Mak, R. Renneberg, W. Sterry, and A. Patzelt. Drug delivery with topically applied nanoparticles: Science fiction or reality? Skin Pharmacology and Physiology, 26(46):227-233, 2013.

[2] J. Lademann, F. Knorr, H. Richter, S. Jung, M. C. Meinke, E. Rühl, U. Alexiev, M. Calderon, and A. Patzelt. Hair follicles as a target structure for nanoparticles. Journal of Innovative Optical Health Sciences, 08(04):1530004, July 2015.

[3] Tarl W. Prow, Jeffrey E. Grice, Lynlee L. Lin, Rokhaya Faye, Margaret Butler, Wolfgang Becker, Elisabeth M. T. Wurm, Corinne Yoong, Thomas A. Robertson, H. Peter Soyer, and Michael S. Roberts. Nanoparticles and microparticles for skin drug delivery. Advanced Drug Delivery Reviews, 63(6):470-491, May 2011.

[4] Juergen Lademann, Heike Richter, Alexa Teichmann, Nina Otberg, Ulrike BlumePeytavi, Javiana Luengo, Barbara Weiss, Ulrich F. Schaefer, Claus-Michael Lehr, Roger Wepf, and Wolfram Sterry. Nanoparticles-an efficient carrier for drug delivery into the hair follicles. Eur J Pharm Biopharm, 66(2):159-164, May 2007. PMID: 17169540 .

[5] Annika Vogt, Behazine Combadiere, Sabrina Hadam, Karola M. Stieler, Juergen Lademann, Hans Schaefer, Brigitte Autran, Wolfram Sterry, and Ulrike BlumePeytavi. $40 \mathrm{~nm}$, but not 750 or 1,500 nm, nanoparticles enter epidermal CD1a+ cells after transcutaneous application on human skin. Journal of Investigative Dermatology, 126(6):1316-1322, June 2006.

[6] Hagar I. Labouta and Marc Schneider. Interaction of inorganic nanoparticles with the skin barrier: current status and critical review. Nanomedicine, 9(1):39-54, January 2013. PMID: 22542824. 
[7] R. Alvarez-Román, A. Naik, Y. N. Kalia, R. H. Guy, and H. Fessi. Skin penetration and distribution of polymeric nanoparticles. Journal of Controlled Release, 99(1):5362, September 2004.

[8] U. Blume-Peytavi and A. Vogt. Human hair follicle: reservoir function and selective targeting. British Journal of Dermatology, 165:13-17, October 2011.

[9] Juergen Lademann, Alexa Patzelt, Heike Richter, Christina Antoniou, Wolfram Sterry, and Fanny Knorr. Determination of the cuticula thickness of human and porcine hairs and their potential influence on the penetration of nanoparticles into the hair follicles. J. Biomed. Opt, 14(2):021014-021014-4, 2009.

[10] Alexa Patzelt, Heike Richter, Fanny Knorr, Ulrich Schäfer, Claus-Michael Lehr, Lars Dähne, Wolfram Sterry, and Juergen Lademann. Selective follicular targeting by modification of the particle sizes. Journal of Controlled Release, 150(1):45-48, February 2011.

[11] Alexa Patzelt, Heike Richter, Lars Dähne, Peter Walden, Karl-Heinz Wiesmüller, Ute Wank, Wolfram Sterry, and Jürgen Lademann. Influence of the vehicle on the penetration of particles into hair follicles. Pharmaceutics, 3(2):307-314, June 2011. PMID: 24310497 PMCID: PMC3864236.

[12] R. Dean Astumian. Thermodynamics and kinetics of a brownian motor. Science, 276(5314):917-922, May 1997.

[13] Frank Jülicher, Armand Ajdari, and Jacques Prost. Modeling molecular motors. Rev. Mod. Phys., 69(4):1269-1282, October 1997.

[14] Peter Reimann. Brownian motors: noisy transport far from equilibrium. Physics Reports, 361(2-4):57-265, April 2002.

[15] Peter Hänggi and Fabio Marchesoni. Artificial brownian motors: Controlling transport on the nanoscale. Rev. Mod. Phys., 81(1):387-442, March 2009.

[16] Kazuo Kitamura, Makio Tokunaga, Atsuko Hikikoshi Iwane, and Toshio Yanagida. A single myosin head moves along an actin filament with regular steps of 5.3 nanometres. Nature, 397(6715):129-134, January 1999.

[17] I.M. Kulic, M. Mani, H. Mohrbach, R. Thaokar, and L. Mahadevan. Botanical ratchets. Proceedings of the Royal Society B: Biological Sciences, 276(1665):22432247, June 2009.

[18] M. Ossadnik, H. Richter, A. Teichmann, S. Koch, U. Schäfer, R. Wepf, W. Sterry, and J. Lademann. Investigation of differences in follicular penetration of particleand nonparticle-containing emulsions by laser scanning microscopy. Laser Physics, 16(5):747-750, 2006.

[19] Roland Bartussek, Peter Hänggi, and Jürgen G. Kissner. Periodically rocked thermal ratchets. EPL (Europhysics Letters), 28(7):459, 1994.

[20] Donald L. Ermak and J. A. McCammon. Brownian dynamics with hydrodynamic interactions. J. Chem. Phys., 69(4):1352, 1978. 
[21] E. O. Butcher and A. Coonin. The physical properties of human sebum. J. Invest. Dermatol., 12(4):249-254, April 1949. PMID: 18120703.

[22] M. Ito. The innermost cell layer of the outer root sheath in anagen hair follicle: Light and electron microscopic study. Archives of Dermatological Research, 279(2):112-119, December 1986.

[23] V. Kharchenko and I. Goychuk. Flashing subdiffusive ratchets in viscoelastic media. New Journal of Physics, 14:043042, 2012.

[24] V. Kharchenko and I. Goychuk. How molecular motors work in the crowded environment of living cells: Coexistence and efficiency of normal and anomalous transport. Plos One, 9:e91700, 2014. 